\title{
Polarized Helium to Image the Lung
}

\author{
Michèle Leduc and Pierre Jean Nacher \\ leduc@lkb.ens.fr - nacher@lkb.ens.fr \\ Laboratoire Kastler Brossel, ENS, 24 rue Lhomond, F75005 Paris ${ }^{1}$
}

\begin{abstract}
The main findings of the european PHIL project (Polarised Helium to Image the Lung) are reported. State of the art optical pumping techniques for polarising ${ }^{3} \mathrm{He}$ gas are described. MRI methodological improvements allow dynamical ventilation images with a good resolution, ultimately limited by gas diffusion. Diffusion imaging appears as a robust method of lung diagnosis. A discussion of the potential advantage of low field MRI is presented. Selected PHIL results for emphysema are given, with the perspectives that this joint work opens up for the future of respiratory medicine.
\end{abstract}

\section{Introduction}

In 1994 first images of Magnetic Resonance Imaging (MRI) were published showing pulmonary cavities of a dead mouse, inflated with optically polarised ${ }^{129} \mathrm{Xe}$ gas [1]. Soon after, research teams in the USA and in Germany, associating atomic and MRI physicists with radiologists, demonstrated the possibility to image human lung airways using inhaled ${ }^{3} \mathrm{He}[2,3]$.

These gases are chemically inert, non radioactive noble gases, both of nuclear spin $1 / 2$. They are spin polarised prior to inhalation by optical pumping methods, which means that a large non equilibrium magnetization is obtained by orientation of the magnetic moments of the gas nuclei. Then the gas is inhaled in the lungs and imaged by MRI in conventional scanners tuned to the NMR frequency of the gas atoms. This method allows visualising the intrapulmonary airspaces filled with the gas, instead of the tissues examined by conventional proton MRI. The large polarisation of the gas (up to 80\%) compensates for the lower density of nuclear spins in the gas.

Images of the gas in the lungs can thus be recorded during a breathhold with a high resolution. Furthermore, the MRI acquisition sequences can be fast enough to provide dynamical ventilation data. Mapping physical parameters such as local relaxation times or apparent diffusion coefficients (ADC) indirectly provide information on the lung function or may reveal subtle changes in the microstructure of the airways. It is generally recognized that this new imaging method has unprecedented potential for morphological and functional analysis of the lung. It is expected to be a novel addition to the arsenal of pulmonary diagnostic tests and could enable the development of MRI in the chest, traditionally hampered by the low number density of protons and the magnetic field inhomogeneities that exist at the air-tissues boundaries in the lung.

\footnotetext{
${ }^{1}$ Laboratoire Kastler Brossel is a unité de recherche de l'Ecole Normale Supérieure et de l'Université Pierre et Marie Curie, associée au CNRS (UMR 8552).
} 


\section{PHIL: a European consortium to study emphysema}

It was decided in 2000 to put forward a European consortium, coordinated by one of the authors (M.L.), to link the efforts of nine teams in five different countries. Participants were selected for their pre-existing know-how and complementary competences in atomic physics (Paris, Mainz and Krakow), MRI methodology and instrumentation (Orsay and Lyon), radiology and respiratory medicine (Mainz, Sheffield and Copenhagen) and animal model studies (Madrid and Lyon).

The PHIL consortium ("Polarised Helium to Image the Lung", 4]) joined efforts to demonstrate the potential and the validity of the new non invasive method as a diagnostic and prognostic tool for given lung pathologies: emphysema and selected Chronical Obstructive Pulmonary Diseases (COPD), such as bronchitis and bronchiolitis. This choice was motivated by the frequent occurrence of these diseases and the very high cost of their treatment for society: $10 \%$ of the population and $25 \%$ of the smokers suffer from COPD, which is the fourth cause of mortality in Europe. The core of the project was to perform a clinical trial on a large group of patients with the ${ }^{3} \mathrm{He}$ MRI method and with conventional techniques: pulmonary function tests, High Resolution Computed Tomography (HRCT), Krypton scintigraphy. An important objective of the PHIL project was to provide new tools for the study of COPD, aiming at differentiation of various types of diseases, as well as their detection at an early stage, with expectation that in the long range the findings of the project could lead to monitoring therapeutic treatment.

\section{Polarising ${ }^{3} \mathrm{He}$ by metastability exchange optical pumping}

The only candidates as inhaled gaseous tracers are ${ }^{3} \mathrm{He}$ and ${ }^{129} \mathrm{Xe}$, which are spin 1/2 noble gas atoms (isotopes with higher nuclear spins are disregarded, because they are too sensitive to nuclear relaxation processes). ${ }^{3} \mathrm{He}$ has been chosen for several reasons: It provides much larger NMR signals, as its magnetic moment is nearly three times larger than that of ${ }^{129} \mathrm{Xe}$. The ${ }^{3} \mathrm{He}$ atoms can be polarised in larger quantities and to higher polarisation rates. Helium does not cross the alveolar air/blood barrier and remains confined within the air spaces. It is absolutely harmless and induces no side effects, whereas xenon is known as a potent anaesthetic at high concentrations.

${ }^{3} \mathrm{He}$ can be polarised by two established methods which rely on optical pumping techniques. Optical pumping is a very efficient method to control the atomic spin state through interaction with a resonant light beam carrying angular momentum. In the presence of a magnetic guiding field $B$ and of weak relaxation processes, the net result of the repeated light absorption and re-emission cycles is a change in the relative populations of the involved atomic sublevels, i.e. creation of spin orientation. For polarising ${ }^{3} \mathrm{He}$, two routes are possible: spin exchange optical pumping (SEOP) [5], with indirect transfer of angular momentum from a polarised laser beam to the ${ }^{3} \mathrm{He}$ nuclei via alkali metal atoms 
(usually rubidium), and metastability exchange optical pumping (MEOP) 6], with direct transfer of angular momentum from resonant laser light to ${ }^{3} \mathrm{He}$ atoms. Each technique has its own advantages and limitations. For instance, SEOP directly operates at high pressure but is a slow process (several hours are required to polarise helium), while MEOP is a much faster process (it only takes seconds to polarise $100 \mathrm{~cm}^{3}$ of gas at $1 \mathrm{mbar}$ ), but only operates at low pressure ( $\sim$ mbar $)$ and requires further compression for its application to lung MRI. SEOP has been used for several imaging experiments [7], but MEOP has been preferred for human lung imaging in the PHIL project because of its faster production rates and higher nuclear polarisations.

The principle of a ${ }^{3} \mathrm{He}$ optical pumping experiment using MEOP is illustrated in figure 1. A fraction of the helium atoms are excited by a plasma discharge to

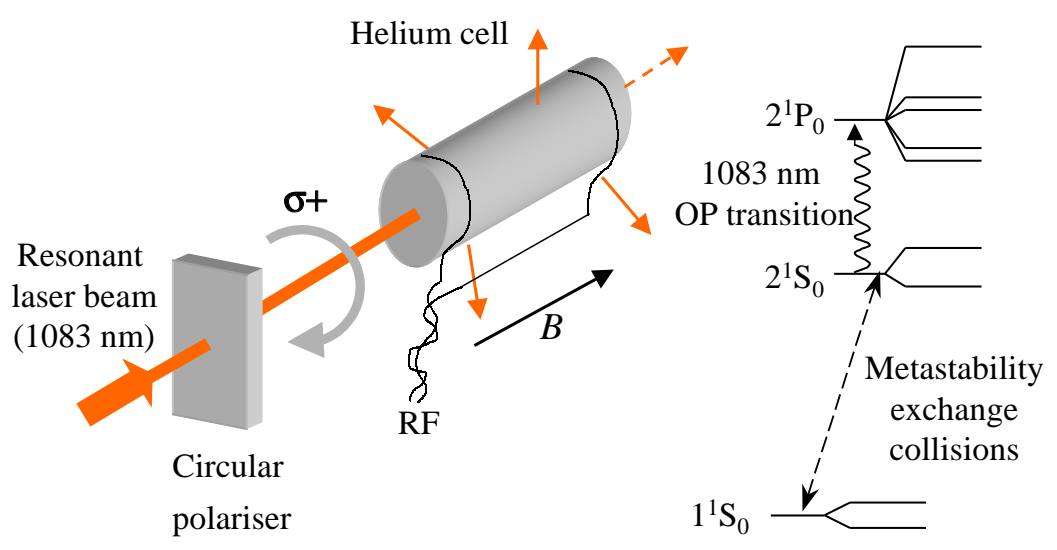

Figure 1: Left: schematic view of an optical pumping setup. In a low-pressure helium-3 gas cell, a weak RF discharge promotes a small fraction $\left(\sim 10^{-6}\right)$ of the atoms into the excited metastable state $2^{3} \mathrm{~S}$, where resonant absorption of the circularly polarised $1083 \mathrm{~nm}$ light can occur. Repeated cycles of absorption/emission of photons transfer a substantial polarisation in the $2^{3} \mathrm{~S}$ state. Nuclear polarisation is transferred to the ground state atoms by metastability exchange collisions. Right: atomic levels of helium-3 and physical processes involved in the OP cycle.

a metastable energy state, where they can absorb resonant light to be optically pumped. For the ${ }^{3} \mathrm{He}$ isotope in this excited metastable state, an efficient coupling between the nucleus and the electrons (the hyperfine interaction) results in a strong entanglement of electronic and nuclear spins. Therefore optical orientation of the electronic angular momentum simultaneously induces nuclear orientation as well. This nuclear orientation is rapidly transferred to the atoms having remained in the ground state through metastability exchange collisions. This process corresponds to a very short interaction between an excited atom and a ground state atom, which results in a fast exchange of the electronic excitations of the colliding atoms with no change in the nuclear orientations. 
In actual gas polarisation devices, a uniform magnetic field (of order $1 \mathrm{mT}$ ) is applied over the whole setup to prevent magnetic relaxation. Powerful fibre lasers at $1083 \mathrm{~nm}$ are used for efficient optical pumping 8. MEOP-based gas polarisation, which requires compression of the polarised gas, was developed for PHIL following two routes with complementary advantages:

- Centralised production of very high grade hyperpolarised gas and transport to the MRI centre. The Mainz group pioneered the development of dedicated systems for massive production of hyperpolarised ${ }^{3} \mathrm{He}$ gas [9]. Sophisticated piston compressors lead to unrivalled performance in terms of output pressure, gas polarisation and production rate. Such facilities deliver remarkably large flux (tens of bar litres per day) of high grade polarised ${ }^{3} \mathrm{He}$ : over $80 \%$ at the production unit, still reaching $60 \%$ after shipping by air (from Mainz to Copenhagen or to Sheffield) 10. The transportation problems were overcome using special containers preventing magnetic relaxation and appropriate ironfree glass for the storage cells containing the gas.

- On-site production of gas with compact polarisers implemented next to the MRI scanner. The delivered gas may be of lower grade $\left({ }^{3} \mathrm{He}\right.$ is diluted in a neutral gas like nitrogen, or produced with lower polarisation, of order $40 \%$ ), but problems with storage and transportation are avoided. The key element of the system developed by the Paris group is a compact polarisation-preserving peristaltic compressor that allows production of polarised gas next to or inside the MRI scanner [11]. Two different prototypes of such compact and robust polarisers were built, and are ready for possible commercial production.

\section{Pulmonary ventilation images: resolution and dynamics}

Figure 2 displays chest MRI images obtained at 1.5 Tesla with conventional proton MRI (left) and polarised helium MRI (right). On the proton scan, most
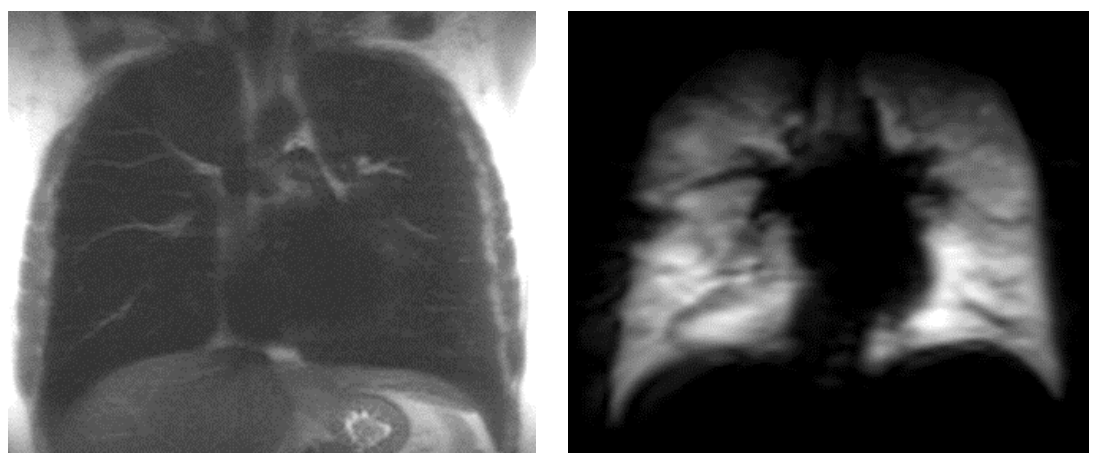

Figure 2: Chest MR images (1 $\mathrm{cm}$ thick slices) of the same normal subject. Left: fast spin-echo proton image. Right: FLASH ${ }^{3} \mathrm{He}$ image of the gas in the lungs. The NMR frequency is changed from $64 \mathrm{MHz}$ for protons to $49 \mathrm{MHz}$ for ${ }^{3}$ He. Courtesy of J. Wild, Academic Radiology dept., U. of Sheffield. 
tissues are visible, but the lung parenchyma does not appear: this is due partly to the low density of the lung tissues, partly to the shortening of the transverse relaxation time $T_{2}$ by the non-uniform magnetisation of the inhomogeneous parenchyma in the high magnetic field of the scanner. On the helium scan, the gas-filled pulmonary air spaces are the only visible part of the chest. Shortening of $T_{2}$ by susceptibility effects is less severe thanks to the rapid gas diffusion at the alveolar scale, a well known motional averaging and line narrowing effect. A set of 15-20 such slices at adjacent positions in the chest allows re-constructing a 3D map of gas distribution in the lungs.

Another attractive possibility offered by the ${ }^{3} \mathrm{He}-\mathrm{MRI}$ technique is access to dynamical parameters of the lung ventilation. A typical temporal resolution of order $0.1 \mathrm{~s}$ is required to monitor the respiratory cycle. For ultra-fast imaging, a radial acquisition strategy combined with a sliding window method was developed. This method, referred as SPIRO (Sliding Pulmonary Imaging for Respiratory Overview), allows a regional quantification of dynamical ventilation parameters using a single breath of polarised gas. Figure 3 shows two series of helium lung images (here 2D projections) taken during a respiratory cycle. They show the gas flowing first down the trachea, then into the bronchi
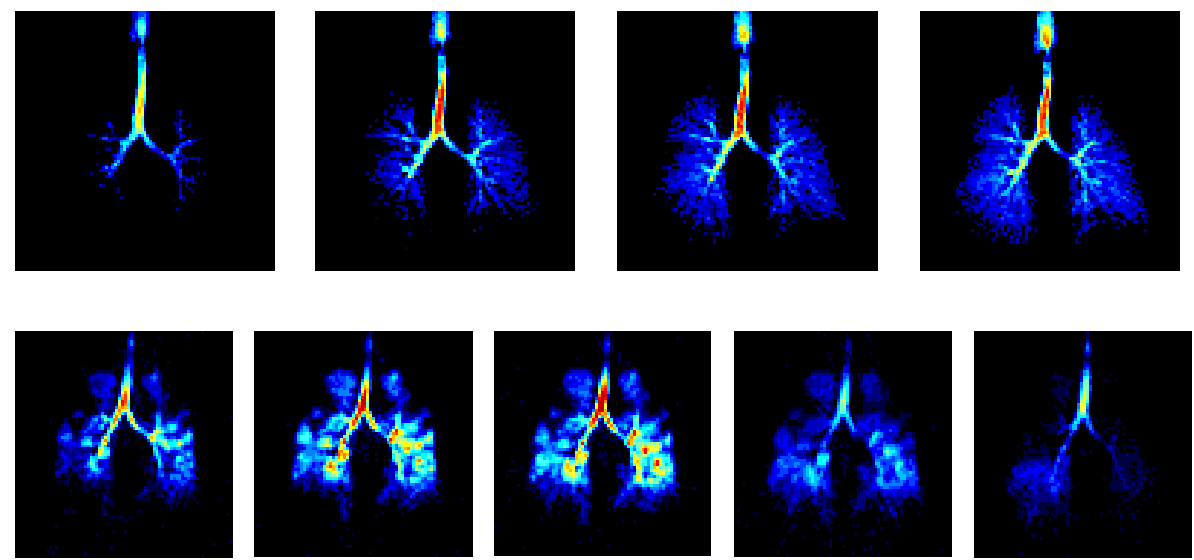

Figure 3: Dynamic images collected using a sliding window radial sequence [12; the time interval between displayed images is $0.5 \mathrm{~s}$. Top row: healthy normal subject, from the first part of an inhalation of $300 \mathrm{ml}$ of ${ }^{3} \mathrm{He}$ polarized to $40 \%$. Bottom row: COPD patient showing regions of ventilation obstruction in both lungs, particularly in the upper lobes, and a delayed emptying/depolarization of gas in the lower left lobe which could be indicative of air trapping. Courtesy of J. Wild, Academic Radiology dept., U. of Sheffield.

and finally in the peripheral lung. Clear differences are visible between a normal subject (top row) and a patient suffering from COPD (bottom row), with wedge-shaped defects indicating that some pulmonary zones are poorly ventilated. Key functional parameters can be extracted from the images, such as 
gas arrival time, filling time constant, and gas volume. Regional maps of such parameters can be used to assess the degree of the disease severity.

The resolution of these helium images, even if superior to that of competing techniques, is however intrinsically limited and does not allow visualising the airways at the scale of the alveoli (of order $0.1 \mathrm{~mm}$ ). Proton MRI can reach micrometric resolution for small objects if sufficient signal to noise ratio is achieved. The same resolution is not possible for helium MRI, due to the rapid diffusion of the gas inside the airways. MRI relies on frequency encoding of the position through the use of magnetic field gradients, sequentially applied along various directions in space. If $G$ is a field gradient applied along the $x$ direction, the NMR frequency change across a distance $\delta x$ is $\delta f=\gamma G \delta x(\gamma=32$ $\mathrm{MHz} / \mathrm{T}$ is the gyromagnetic ratio of ${ }^{3} \mathrm{He}$ ). To resolve details of size $\delta x, G$ must be applied for a time $\tau$ such that $\tau \delta f \sim 1$, hence $\delta x \sim 1 / \gamma G \tau$. When dealing with a gas, the observation time $\tau$ is actually limited by the diffusion of the gas over $\delta x$, namely $\tau \sim \delta x^{2} / D\left(D \sim 80 \mathrm{~mm}^{2} / \mathrm{s}\right.$ is the diffusion coefficient of ${ }^{3} \mathrm{He}$ in air). Thus the resolution is limited to $\delta x \sim(D / \gamma G)^{1 / 3}$, of order $0.4 \mathrm{~mm}$ for a conventional scanner with typical gradients reaching $0.3 \mathrm{mT} / \mathrm{cm}$.

\section{Apparent Diffusion Coefficient}

In spite of the limitations on image resolution with ${ }^{3} \mathrm{He}-\mathrm{MRI}$, measurements of apparent diffusion coefficients (ADC) can provide relevant information on the lung microstructure. When diffusing freely in air (in the absence of restricting walls or barriers), helium atoms are displaced on average by an amount $\sqrt{D \tau}$, e.g. $0.9 \mathrm{~mm}$ after a time $\tau=10 \mathrm{~ms}$. In the lung, free diffusion occurs at such time scales only in larger airways, while collisions with the walls of smaller airways and of alveoli reduce displacements of helium atoms. A time- and scaledependent ADC is introduced to characterise this restricted diffusion. It is not easy to relate this reduced value to a detailed description of the microstructure of the lung at the alveolar level due to its complexity. However measuring ADC values can be useful to assess pathological changes. For instance in emphysema, the restrictions to diffusion are reduced due to expansion of alveoli and modification of connectivity between them, which results in an increased ADC.

Several NMR techniques can be used to measure diffusion coefficients. They rely on applying a sensitising gradient to imprint a non-uniform magnetisation pattern during a given time, and on measuring the resulting magnetisation decay. The ratio of images recorded with and without such a diffusion-sensitising gradient is used to compute a diffusion map. Figure 4 displays ADC maps obtained in the lung with a standard technique (diffusion weighting by a bipolar gradient pair). One observes an increase by more than a factor two for the mean $\mathrm{ADC}$ value for a COPD patient compared to a normal subject. One also notes a significant difference between the ADC histograms recorded for an asymptomatic smoker and a normal subject; this indicates that ADC measurements have a potential for detection of mild COPD diseases. 

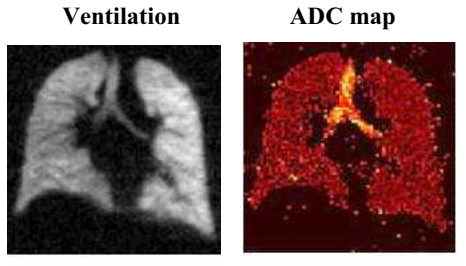

ADC Histogram $\left(\mathrm{cm}^{2} / \mathrm{s}\right)$
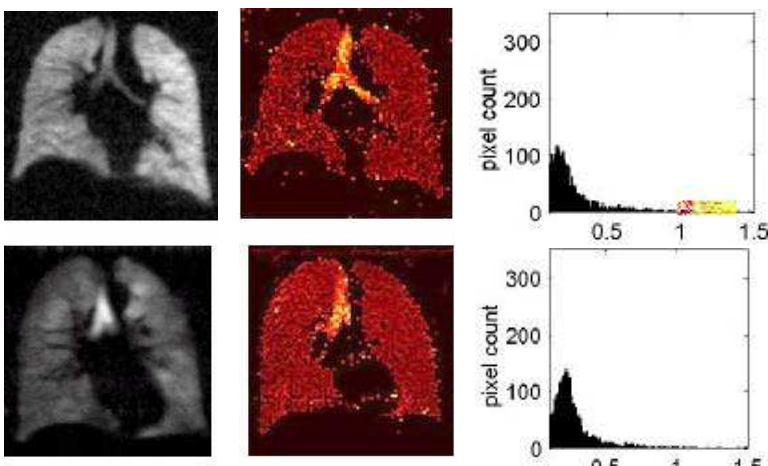

Normal
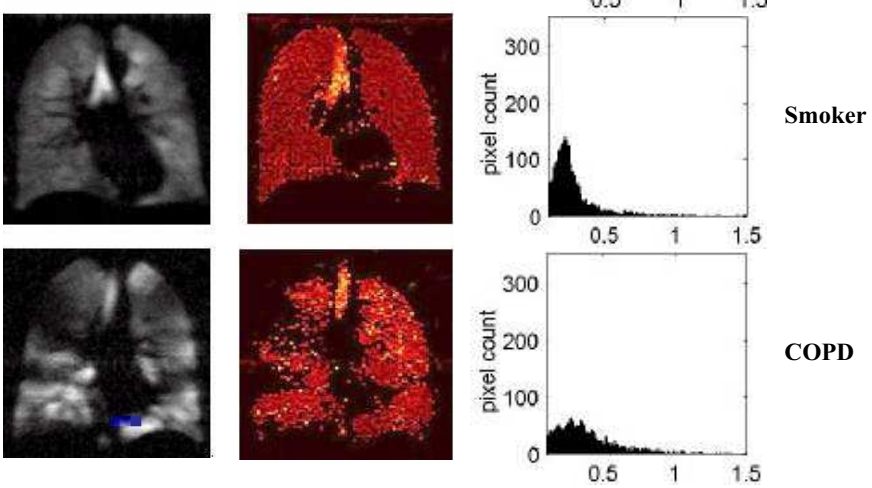

Figure 4: Left to right: gas distribution images, ADC maps and ADC histograms in 3 subjects (top to bottom). Courtesy of J. Wild, Academic Radiology dept., U. of Sheffield.

\section{Low field versus high field for ${ }^{3} \mathrm{He}$ MRI?}

In conventional proton MRI, high magnetic fields are used to obtain sufficient equilibrium proton magnetisation and NMR signal. Noise arises from thermal (Johnson) noise in the patient's tissues and in the NMR receiving coil. Usually, the former noise source dominates; for a given magnetisation, both the NMR signal and the noise are proportional to frequency (i.e. to the field). For ${ }^{3} \mathrm{He}-$ MRI with optically pumped gas, the magnetisation of the gas and hence the signal to noise ratio (SNR) do not depend on the field (this contrasts with the usual proton MRI for which the equilibrium magnetisation, and hence the SNR, increase linearly with the field). However this breaks down at very low field (below about $30 \mathrm{mT}$, depending on the coil size and technology), where the patient noise decreases below the thermal noise of the coil.

The PHIL Partner in Orsay demonstrated that there is no SNR penalty when using a low field MRI scanner at $0.1 \mathrm{~T}[13]$. On the contrary, there is an important benefit of low-field operation: with reduced gradients arising from the magnetic susceptibility of tissues, transverse relaxation times are much longer at $0.1 \mathrm{~T}$ than at $1.5 \mathrm{~T}$. Weaker field gradients can then be used for imaging or ADC measurements. This allows measuring diffusion coefficients at longer time scales, thus providing more information on the connectivity of lung air spaces. A precise way of measuring ADC coefficients in this case is through the decay of spin echo trains. 
However, shortening of transverse relaxation times can also be viewed as a useful contrast mechanism since it depends on the lung microstructure. It was for instance demonstrated to be more sensitive to the degree of lung inflation at $1.5 \mathrm{~T}$ than at $0.1 \mathrm{~T}[14$.

A clear advantage of operating at low field is the reduced cost and increased flexibility of the imaging system: an open geometry can be designed, and standing or sitting patients may be examined. Studies at ultra low field in a home made vertical scanner at $3 \mathrm{mT}$ have started with the PHIL Partner in Paris 15. This could open up possibilities of low-cost dedicated scanners for the screening or follow-up of lung diseases.

\section{Emphysema studies with ${ }^{3} \mathrm{He}$ MRI}

The PHIL multicentre clinical trial, after upgrading the hardware and software of the involved MRI scanners according to the ${ }^{3} \mathrm{He}$ MRI standards, put forward a standardized protocol to study a significant number of patients and healthy volunteers for reference. Pre-existing sequences initially used in Mainz were adapted and optimized at the different sites: ventilation distribution (breathhold), apparent diffusion coefficient (breath hold) and dynamic ventilation (single respiratory cycle). The protocol also included pulmonary function tests to assess severity scores. Data were recorded in a data base and finally submitted to a statistical analysis, which provided a wealth of information, still not fully exploited [16.

The subjects were divided in three groups: 79 patients with proven COPD, among whom 17 suffered from alpha-1-ATD (anti-trypsin deficiency) and 37 healthy volunteers. A score number referring to the degree of abnormality was attributed to each subject. Comparison between HRCT, MRI findings and abnormality scores showed that MRI correlates better with pulmonary function tests than HRCT. The vast majority of MRI findings consisted of wedged shaped defects, even subjects with normal HRCT showed ventilation defects. The comparison of ADC with emphysema index and mean lung density was also exploited. ADC measurements clearly separated the healthy subjects from those with emphysema of any type. ${ }^{3} \mathrm{He}$ MRI proved also to be able to distinguish between different diseases by using a combination of ventilation distribution and ADC mapping. After assessing all the results, one general finding is that HRCT is usually more sensitive but MRI is more specific. A positive feature of the ${ }^{3} \mathrm{He}$ MRI method is that a single exam provides several unique complementary informations at the same time (morphology, ventilation defects, average of air spaces, even in-vivo oxygen concentration and uptake that can be derived from the local relaxation times).

The animal models studies of PHIL provided very complementary results [17. Different models of lung diseases were induced in rats with chemicals such as elastase or cadmium chloride. To some extent these models mimic human emphysema. The rat groups treated with cadmium showed clear ventilation defects, corresponding to non-ventilated areas of the partially collapsed lung. 
ADC and post-mortem morphometric measurements were compared. The most significant finding of this work is the excellent correlation between the ADC data and the morphometric ones, which allow direct quantification of alveolar size. Complementary to experiments in clinical trials, in which the available population generally have suffered chronic diseases, emphysema in animals was mildly developed to evaluate the technique for early diagnosis. In that perspective, low field scanners could be highly useful, as the system successfully built in Krakow with a permanent magnet of $0.08 \mathrm{~T}$ based on a permanent magnetic material of new generation. Such low cost scanners are adapted to imaging small animal lungs and may be especially valuable for pharmaceutical research.

\section{Conclusion}

In this multidisciplinary work, atomic physicists played a prominent role at the early stage of the technique. The ${ }^{3} \mathrm{He}$ polarisation methods were first developed in the 80's for the needs of polarised quantum fluids physics, for polarised targets in nuclear physics and more recently for neutron spin filters at reactors. Finally, in view of application to MRI, the metastability exchange optical pumping method was pushed to its limits and very appropriate polarisers were constructed by the PHIL consortium. They bring a satisfactory solution for clinical implementation of ${ }^{3} \mathrm{He} \mathrm{MRI}$, either by remote production of high grade gas or in-situ table-top polarisation. There is still room for improvement of fast dedicated MRI sequences and signal to noise enhancement. Great expectation is also related to dedicated low field scanners, with possible extension of the ${ }^{3} \mathrm{He}$ MRI lung imaging method to developing countries.

The PHIL consortium has shown that ${ }^{3} \mathrm{He}$ MRI can be routinely performed on a significant number of patients and shows interesting new insights into common lung diseases, COPD and emphysema. It proves superior in attaining $3 \mathrm{D}$ lung function in the lungs, and is more closely correlated with routine lung function tests than HRCT. The method has already been tested in other lung diseases, and more studies are planned or have started. These include lung transplant assessment, cystic fibrosis (the most common genetic lung disease), asthma and lung cancer. The technology has proved of huge potential interest in these areas, because it is non-invasive and does not require ionising radiation, an extremely important feature, especially in longitudinal follow-up of children. It could also be very beneficial for the development of novel therapies, reducing the size of trials and the number of sacrificed animals.

\section{References}

[1] M.S. Albert, G.D. Cates, B. Driehuys et al., Nature 370, 199 (1994): Biological MRI using laser-polarized ${ }^{129} \mathrm{Xe}$

[2] J.R. MacFall, H.C. Charles, R.D. Black et al., Radiology 200, 553 (1996): Human lung air spaces: potential for MRI with hyperpolarized ${ }^{3} \mathrm{He}$ 
[3] H.U. Kauczor, D. Hofmann, K.F. Kreitner et al., Radiology 201, 564 (1996): Normal and abnormal pulmonary ventilation: Visualization at hyperpolarized ${ }^{3} \mathrm{He} \mathrm{MRI}$

[4] cf. http:// www.phil.ens.fr. The PHIL project has been supported by the EC contract QLG1-2000-01559 running from 12/2000 to 06/2004.

[5] T.G. Walker and W. Happer, Rev. Mod. Phys. 69, 629 (1997): Spinexchange optical pumping of noble-gas nuclei

[6] P.-J. Nacher and M. Leduc, J. Physique 46, 2057 (1985): Optical pumping in ${ }^{3} \mathrm{He}$ with a laser

[7] T. Chupp and S. Swanson, Adv. At. Mol. Opt. Phys. 45, 51 (2001): Medical imaging with laser polarised noble gases

[8] G. Tastevin, S. Grot, E. Courtade et al., Appl. Phys. B 78, 145 (2004): A broadband $\mathrm{Yb}$-doped tunable fiber laser for He optical pumping at $1083 \mathrm{~nm}$

[9] J. Becker, J. Bermuth, M. Ebert et al., Nucl. Instr. Meth. Phys. Res. 402, 327 (1998): Interdisciplinary experiments with polarized ${ }^{3} \mathrm{He}$

[10] E.J.R Van Beek, J. Schmiedeskamp, J.M. Wild et al., Eur. Radiol. 13, 2583 (2003): Hyperpolarized ${ }^{3} \mathrm{He}$ MRI of the lungs: improving accessibility to gas using a central production facility and transport by air

[11] P.-J. Nacher, G. Tastevin, X. Maitre et al., Eur. Radiol. 9 B18 (1999): A peristaltic compressor for hyperpolarised helium

[12] J. Wild, M.N.J. Paley, L. Kasuboski et al., Magn. Reson. Med. 49, 991 (2003): Dynamic radial projection MRI of inhaled hyperpolarized ${ }^{3} \mathrm{He}$

[13] E. Durand, G. Guillot, L. Darrasse et al., Magn. Reson. Med. 47, (2002) 75: CPMG measurements and ultrafast imaging in human lungs with hyperpolarized ${ }^{3} \mathrm{He}$ at low field $(0.1 \mathrm{~T})$

[14] L. de Rochefort, A. Vignaud, X. Maitre et al., Proc. 12th ISMRM meeting ISSN 1545-4436, 2724 (2004): Influence of lung filling on $\mathrm{T}_{2}{ }^{*}$ values in human at $1.5 \mathrm{~T}$ with hyperpolarised ${ }^{3} \mathrm{He}$

[15] C.P. Bidinosti, J. Choukeife, G. Tastevin et al., Magnetic Resonance Materials in Physics, Biology, and Medicine (MAGMA) 16, 255 (2004): MRI of the lung using hyperpolarized ${ }^{3} \mathrm{He}$ at very low magnetic field $(3 \mathrm{mT})$

[16] K.K. Gast, A. Dahmen, C.P. Heussel et al., Proc. 12th ISMRM meeting ISSN 1545-4436, 1673 (2004): ${ }^{3} \mathrm{He}-\mathrm{MRI}$ in a european multicenter trial in COPD and emphysema PHIL

[17] G. Peces-Barba, J. Ruiz-Cabello, Y. Cremillieux et al., Eur. Resp. J. 22, 14-19 (2003): ${ }^{3} \mathrm{He}$ diffusion coeficient: correlation to morphometry in a model of mild emphysema 